\title{
Diethyl 2,6-Dimethyl,4-(1,1,1-trichloromethyl)-1,4-dihydropyridine-3,5-dicarboxylate
}

\section{Fatma Aydin ${ }^{a} *$ and Recep Ozen}

${ }^{a}$ Canakkale Onsekiz Mart University, Department of Chemistry, 17020 Canakkale TURKEY

${ }^{\mathrm{b}}$ Mersin University, Department of Chemistry, 33342 Mersin TURKEY

Tel: +90 286 2180018-1862, Fax: +902862180533 e-mail: faydin@comu.edu.tr

*Author to whom correspondence should be addressed

Received: 12 October 2005 / Accepted: 24 October 2005 / Published: 28 February 2006

Keywords: Hantzsch reaction, 1,4-dihydropyridines, chloral, $\mathrm{KF} / \mathrm{Al}_{2} \mathrm{O}_{3}$

In 1882, Hantzsch reported the first synthesis of dialkyl 1,4-dihydro-2,6-dimethylpyridine3,5-dicarboxylates from a refluxing mixture of an aldehyde, a $\beta$-ketoester, and aqueous ammonium hydroxide in ethanol.[1] 1,4-dihydropyridines (1,4-DHPs) are well known as $\mathrm{Ca}^{+2}$ channel blockers.[2,3]

\section{$2 \mathrm{CH}_{3} \mathrm{COCH}_{2} \mathrm{COOC}_{2} \mathrm{H}_{5}+\mathrm{CCl}_{3} \mathrm{CHO}$}

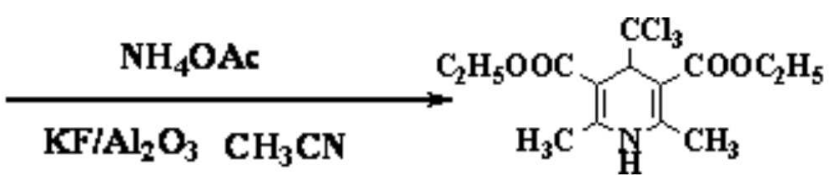

Concentric $\mathrm{H}_{2} \mathrm{SO}_{4}(5 \mathrm{~mL})$ was dropped on chloral hydrate (3 $\mathrm{g}$ ) and 1,1,1-trichloroacetaldehyde was distilled at $98^{\circ} \mathrm{C}$. Acetylacetone $(4 \mathrm{~mL}, 40 \mathrm{mmol})$, freshly distilled 1,1,1-trichloroacetaldehyde ( $2.94 \mathrm{~g}, 20$ mmol) and $\mathrm{CH}_{3} \mathrm{COONH}_{4}(1.44 \mathrm{~g}, 20 \mathrm{mmol})$ were dissolved in acetonitrile $(15 \mathrm{~mL})$. This mixture was added on [4] and refluxed for $3 \mathrm{~h}$. The progress of the reaction was monitored by TLC analysis. After completion of the reaction, the resulting suspension was filtered and solid washed with acetonitrile $(5 \mathrm{~mL})$, solvent was evaporated. Residue was washed with water and extracted in $\mathrm{CH}_{2} \mathrm{Cl}_{2}(3 \times 5 \mathrm{~mL})$. The solution of $\mathrm{CH}_{2} \mathrm{Cl}_{2}$ was dried over anhydrous $\mathrm{Na}_{2} \mathrm{SO}_{4}$ and then filtered. The solvent was removed under reduced pressure and pale yellow oil product in a good yield, $67.1 \%(4.97 \mathrm{~g})$.

UV $\lambda_{\max }(\mathrm{nm}$; ethyl alcohol $) / \varepsilon\left(\mathrm{dm}^{3} \cdot \mathrm{mol}^{-1} \cdot \mathrm{cm}^{-1}\right): 392 / 6270$

IR (KBr) $\left(\mathrm{v} \mathrm{cm}^{-1}\right): 3419(\mathrm{~N}-\mathrm{H}) ; 2984(\mathrm{C}=\mathrm{C}) ; 1722(\mathrm{C}=\mathrm{C}-\mathrm{C}=\mathrm{O}) ; 1031(\mathrm{C}-\mathrm{O}) ; 818(\mathrm{C}-\mathrm{Cl})$.

${ }^{1} \mathrm{H}-\mathrm{NMR}\left(400 \mathrm{MHz}, \mathrm{CDCl}_{3}\right): \delta=1.21\left(\mathrm{t}, 6 \mathrm{H},-\mathrm{CH}_{3}\right) ; 2.16\left(\mathrm{~s}, 6 \mathrm{H},-\mathrm{CH}_{3}\right) ; 3.31(\mathrm{~s}, 1 \mathrm{H}) ; 4.12(\mathrm{q}, 4 \mathrm{H}$, methylenic).

${ }^{13} \mathrm{C}-\mathrm{NMR}\left(100 \mathrm{MHz}, \mathrm{CDCl}_{3}\right): \delta=29.8,31.6,58.5,62.0,81.7,166.7,169.0,199.5$.

Elemental Analysis: Calculated for $\mathrm{C}_{14} \mathrm{H}_{18} \mathrm{Cl}_{3} \mathrm{NO}_{4}$ : C, 45.37\%; H, 4.89\%; Cl, 28.69\%; N, 3.78\%. Found: C, 45.21\%; H, 4.84\%; Cl, 28.72\%; N, 3.82\%.

\section{References}

1. A. Hantzsch, Ann. 215, 1, 72 (1882); Ber. 181744 (1885); 19, 289 (1886).

2. a) Bossert, F.; Meyer, H.; Wehinger, E. Angew. Chem., Int. Ed. Engl. 1981, 20, 762-769;

b) Nakayama, H.; Kasoaka, Y. Heterocycles 1996, 42, 901-909.

3. Love, B.; Goodman, M.; Snader, K.; Tedeschi, R.; Macko, E. J. Med. Chem. 1974, 17, 956-965.

4. Aydin, F. and Ozen, R. J. Chem. Res-S, (7), 2004, 486-487. 
5. Younessi, A. and Krapivin, G. D., Molbank, 2003, M344

5. Younessi, A. and Krapivin, G. D., Molbank
Sample Availability: Available from MDPI.
C $2006 \underline{\text { MDPI. All rights reserved. }}$

5. Younessi, A. and Krapivin, G.
Sample Availability: Available fro
C 2006 MDPI. All rights reserved. 